\title{
Grey Forecasting Models for Social Media Adoptions of Transnational Enterprises
}

\author{
Hsia-Ching Chang \\ CrystalWave Analytics \\ Taipei City, Taiwan \\ e-mail: crystalwave.analytics@gmail.com
}

\author{
Chen-Ya Wang \\ Department of Management \& Information \\ National Open University \\ New Taipei City, Taiwan \\ e-mail: ntuimgrace@gmail.com
}

\begin{abstract}
The lack of adoption data posed difficulties for social media researchers and resulted in scant social media studies focusing on forecasting models. To broach the subject, this study put efforts in studying the benchmark transnational enterprises and collecting the first-hand adoption time data through the APIs of three dominating social media platforms: Youtube, Facebook, and Twitter. Grey system theory facilitates prediction problems of insufficient information, small samples, and uncertainty. Therefore, we compared the performance of two grey forecasting models, GM $(1,1)$ and grey Verhulst model, to gain insight into social media adoption trends. Our preliminary results indicated that GM $(1,1)$ performs better as a potential forecasting model to keep track of trends in adoptions of social media platforms in the market.
\end{abstract}

Keywords-grey system theory; grey forecasting; GM (1,1); social media; grey Verhulst model

\section{INTRODUCTION}

In recent years, a vast majority of businesses and organizations have adopted social media platforms interacting with their customers and connecting potential audience. Benefit from growing mobile Internet access, diverse user-generated content, and huge user bases, Facebook, Twitter, and Youtube have become the dominant social media platforms around the world. Compared to public sectors, transnational enterprises generally stay more sensitive to the trends in the market. Therefore, their adoption decisions and channel choices on social media, combined with the times they adopted the social media platforms, appear to be effective indicators of growth trends. As for the new products or services with short-term historical data of adoptions, applying forecasting models to examine their diffusion and growth trends is relatively challenging. Unlike the Bass model applied to diffusion of innovations (DoI) theory [1] requiring big sample data, grey system theory facilitates in prediction problems with insufficient information, small samples, and uncertain nature. In this study, we draw a sample of 250 Fortune global firms in 2012, collected their adoption times of top three social media platforms, and applied gray forecasting models to take into account the time variable often neglected in the DoI studies. Section 2 begins with relevant literature review. Section 3 deals with the methodology of performing grey forecasting modeling. In Section 4, the modeling results of three adoption time series with GM $(1,1)$ and grey Verhulst model are presented. Finally, Section 5 concludes with implications and future work.

\section{LiTERATURE REVIEW}

Studying DoI or new product lifecycles requires sufficient historical data to identify the diffusion trends and conduct further perditions with forecasting models, such as Bass models, logistic models, and Gompertz models. Few technology forecasting models are available for predicting novel services and products with short-term historical data. Trappey and Wu used both long and short product lifecycle datasets for 22 electronic products to investigate the performance of simple logistic, Gompertz, and the timevarying extended logistic models [2]. In terms of predicting market capacity with limited historical data, they determined that the time-varying extended logistic model they developed outperformed than simple logistic and the Gompertz models in most datasets. However, it might not fit data containing anomalous data points.

Deng developed grey system theory to overcome the forecasting limitations and uncertainty in an effort to make predictions possible based on insufficient data, in particular time series predictions [3]. The minimum of data points required to use grey models is four. Additionally, without restrictions of data distribution, Grey forecasting models not only have been used in a wide variety of domains but also serve as good decision models for making policy recommendations [4]. Grey Verhulst model, one of the evolved grey models, is usually used for comparing the results with GM $(1,1)$ on predictions of the population growth [5], the mobile and fixed broadband [6], and currency exchange market [7]. Real time communication characterizes the timely information social media platforms can quickly disseminate. Since grey predictors are more robust dealing with noise and incomplete information, the capabilities and benefits of applying grey system theory to real time systems have been recognized [7]. The findings of recent studies on and mobile cellular/fix broadband growth [6] and timely currency market [7] seem to confirm the GM $(1,1)$ and the modified GM $(1,1)$ using Fourier series work best in model fitting and forecasting than other grey forecasting models. 


\section{MethodOLOGY}

The fundamental expression of Grey model is GM (m, n) in which $m$ and $n$ refer to the order of differential equation and the number of variables, respectively [4].

\section{A. $\operatorname{GM}(1,1)$}

The simplest Grey Model, namely GM $(1,1)$, presents the first order differential equation and one variable as Eq. (1).

$$
\frac{d X^{(1)}}{d X}+a X^{(1)}=b
$$

However, it only takes non-negative data sequence [4]. The steps of building GM $(1,1)$ model are listed as follows.

1) Obtaining a non-negative time series, $X^{(0)}$, with $n$ observations, and smoothing the series if necessary:

$$
X^{(0)}=\left\{X^{(0)}(1), X^{(0)}(2), \ldots, X^{(0)}(n)\right\}, n \geq 4
$$

However, in many practical situations, we do not know the distribution of $X^{(0)}$ exactly and the series might need to be smoothed or transformed in order to get better modeling results. Therefore, it is essential to check the Eq. (3) and see whether the original series lies in the interval $\left(e^{\frac{-2}{n+1}}, e^{\frac{2}{n+2}}\right)$; otherwise, the procedure for smoothing or transforming the series is required [8].

$$
\lambda(\mathrm{k})=\frac{X^{(0)}(k-1)}{X^{(0)}(k)}, k=2,3, \ldots, n
$$

Frequently used smoothing or transformation techniques include log transformation, moving average, exponential transformation, and so forth.

2) The (first-order) accumulated generating operation (AGO) was carried out and formed as as $X^{(1)}$ :

$$
X^{(1)}=\left\{X^{(1)}(1), X^{(1)}(2), \ldots, X^{(1)}(n)\right\}
$$

3) Generating the mean sequence $Z^{(1)}$ based on consecutive neighbors of $X^{(1)}$ :

$$
Z^{(1)}=\left\{Z^{(1)}(1), Z^{(1)}(2), \ldots, Z^{(1)}(n)\right\}
$$

where $Z^{(1)}$ is given by

$$
Z^{(1)}(\mathrm{k})=0.5 \mathrm{X}^{(1)}(\mathrm{k})+0.5 \mathrm{X}^{(1)}(\mathrm{k}-1), \mathrm{k}=2,3, \ldots, \mathrm{n}
$$

4) Building matrix $B$ and $Y$ for $G M(1,1)$

$$
B=\left(\begin{array}{cc}
-Z^{(1)}(2) & 1 \\
-Z^{(1)}(3) & 1 \\
\ldots & \cdots \\
-Z^{(1)}(\mathrm{n}) & 1
\end{array}\right), \quad Y=\left(\begin{array}{c}
X^{(0)}(2) \\
X^{(0)}(3) \\
\ldots \\
X^{(0)}(\mathrm{n})
\end{array}\right)
$$

5) Solving $\alpha$ consisting of developing coefficient (i.e., a) and influence coefficient (i.e., b) based on ordinary least square methods.

$$
\alpha=\left(\begin{array}{l}
a \\
b
\end{array}\right)=\left(\mathrm{B}^{\mathrm{T}} \mathrm{B}\right)^{-1} \mathrm{~B}^{\mathrm{T}} \mathrm{Y}
$$

6) Estimating the time response sequence of grey prediction model, GM (1,1):

$$
\hat{X}^{(1)}(t+1)=\left[X^{(0)}(1)-\frac{b}{a}\right] e^{-a t}+\frac{b}{a}, t=1,2, \ldots, \mathrm{n}-1
$$

This first-order linear differential equation, called as whitenizing equation, can also be expressed as follows:

$$
\hat{X}^{(1)}(1)=X^{(0)}(1)
$$

7) To predict the value of the next term, we use inverse accumulated generating operation (IAGO):

$$
\hat{X}^{(0)}(\mathrm{t}+1)=\hat{X}^{(1)}(\mathrm{t}+1)-\hat{X}^{(1)}(\mathrm{t}), t=1,2, \ldots, \mathrm{n}-1
$$

If we predict the value at time $(n+k)$, the grey prediction model becomes:

$$
\hat{X}^{(0)}(\mathrm{n}+k)=\hat{X}^{(1)}(\mathrm{n}+k)-\hat{X}^{(1)}(\mathrm{n}+k-1), k \geq 1
$$

\section{B. Grey Verhulst Model (GVM)}

Inspired by the German biologist's Verhulst model, the Grey Verhulst Model was one of the evolved grey models and used to illustrate the series with a S-curve growing pattern and a saturation point. The Grey Verhulst model can be expressed as

$$
\frac{d X^{(1)}}{d X}+a X^{(1)}=b\left(X^{(1)}\right)^{2}
$$

Similar to the GM $(1,1)$ model (i.e., Eq. (1) ), the major difference is the multiplied term $\left(X^{(1)}\right)^{2}$. Additionally, another difference is calculating the inverse accumulated generating operation (IAGO) in the beginning, instead of calculating the (first-order) accumulated generating operation (AGO). Hence, the calculation of matrix B in Eq. (6) would become

$$
B=\left(\begin{array}{cc}
-Z^{(1)}(2) & \left(Z^{(1)}(2)\right)^{2} \\
-Z^{(1)}(3) & \left(Z^{(1)}(3)\right)^{2} \\
\cdots & \ldots \\
-Z^{(1)}(\mathrm{n}) & \left(Z^{(1)}(\mathrm{n})\right)^{2}
\end{array}\right),, Y=\left(\begin{array}{c}
X^{(0)}(2) \\
X^{(0)}(3) \\
\ldots \\
X^{(0)}(\mathrm{n})
\end{array}\right)
$$

We then use the identical equation as GM $(1,1)$, namely Eq. (7), to estimate the grey parameters a and $b$ in order to derive the time response sequence for the GVM as follows:

$$
\hat{X}^{(1)}(t+1)=\frac{a X^{(1)}(0)}{b X^{(1)}(0)+\left[\mathrm{a}-\mathrm{b} X^{(1)}(0)\right] e^{a t}}, t=1,2, \ldots, \mathrm{n}-1
$$

This first-order linear differential equation, called whitenizing equation, can also be expressed as follows:

$$
\hat{X}^{(1)}(1)=X^{(0)}(1)
$$

Likewise, we use the same equation as GM $(1,1)$ model to predict the value at time $(\mathrm{n}+\mathrm{k})$ for the GVM:

$$
\hat{X}^{(0)}(\mathrm{n}+k)=\hat{X}^{(1)}(\mathrm{n}+k)-\hat{X}^{(1)}(\mathrm{n}+k-1), k \geq 1
$$

\section{Performance measures}

The error and the average relative percentage error (ARPE) are common grey forecasting error measures. Additionally, the root-mean-square deviation (RMSD) or root-mean-square error (RMSE) is frequently used to detect the differences between predicted values and actual values. Based on RMSE, the posterior error ratio, c, is calculated to evaluate the accuracy of the model. The equations are listed below.

$$
A R P E=\frac{1}{n-1} \sum_{k=2}^{n} \frac{|\varepsilon(k)|}{X^{(0)}(k)}
$$




$$
c=\frac{S_{2}}{S_{1}}=\frac{\sqrt{\frac{1}{n} \sum_{t=1}^{n}\left(\varepsilon_{t}-\bar{\varepsilon}\right)^{2}}}{\sqrt{\frac{1}{n} \sum_{t=1}^{n}\left(X_{t}^{(0)}-\bar{X}\right)^{2}}}
$$

The corresponding precision rank of different levels of posterior error ratio is organized in Table I [9]:

TABLE I. POSTERIOR ERROR RATIO AND CORESPONDING PRECISION

\begin{tabular}{|l|c|}
\hline \multicolumn{1}{|c|}{ Precision Rank } & Posterior Error Ratio (c) \\
\hline 1 (Highly Accurate) & $\mathrm{c} \leq 0.35$ \\
\hline 2 (Good) & $0.35<\mathrm{c} \leq 0.50$ \\
\hline 3 (Reasonable) & $0.50<\mathrm{c} \leq 0.65$ \\
\hline 4 (Inaccurate) & $0.65<\mathrm{c} \leq 0.80$ \\
\hline
\end{tabular}

The lower the posterior error ratio, the higher precision the forecasting model can achieve. In general, the posterior error ratio below 0.35 is an accurate model and the ratio in between 0.35 and 0.5 is a good model with acceptable accuracy.

\section{Modelling Results}

\section{A. The data}

Based on a random sample of 250 Fortune global 500 firms in 2012, this study identified the social media accounts of those firms disclosed on their global websites and further captured the adoption time data through API platforms of the top three major social media platforms (Youtube, Twitter, and Facebook) from January to February 2013. This study organized three time series into the database and aggregated the yearly time series data of top three social media platform adoptions from 2005 to 2012. Fig. 1 displays the quarterly time series of top 3 social media platform adoptions among the 250 Fortune global firms in between 2005 and 2012.

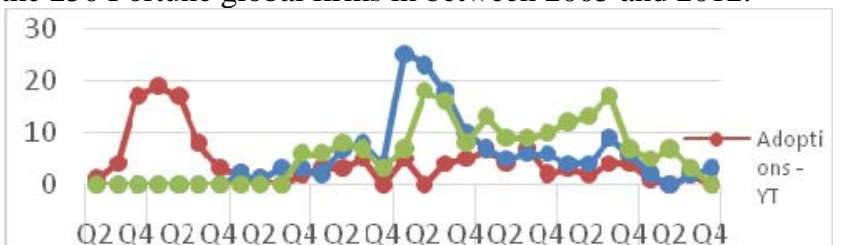

20052006200720082009201020112012

Figure 1. Choices of Adoption Time for the Top Three Social Media Platforms from 2005 to 2012

\section{B. Data analysis and results}

Based on the pre-examination of the yearly adoptions of sampled fortune global firms during 2005 to 2012, we found that our three time series datasets require smoothing operation to reduce the noises. We did log transformation and smoothing; since the modelling results of smoothed datasets outperform the log transformed datasets, we only present the smoothing method here. The smoothing method we applied is three-point moving average. As the following equations, we used the Eq. (16) and Eq. (17) to smooth the first and the last data points in the series, respectively. The data points in between were processed using the Eq. (18) to perform the smoothing.

$$
\begin{aligned}
& X^{(0)}(1)=\left[3 X^{(0)}(1)+X^{(0)}(2)\right] / 4 \\
& X^{(0)}(n)=\left\{X^{(0)}(n-1)+3 X^{(0)}(n)\right] / 4 \\
& X^{(0)}(t)=\left[X^{(0)}(t-1)+2 X^{(0)}(t)+X^{(0)}(t+1)\right] / 4
\end{aligned}
$$

1) Modeling Youtube adoptions of Fortune global firms. Youtube was founded in February 2005. The original Youtube yearly adoption series we collected ranging from 2005 to 2012 is $(22,45,5,11,14,20,13,3)$; the smoothed series using three-point moving average becomes (27.75, $29.25,16.5,10.25,14.75,16.75,12.25,5.5)$. We used the $\mathrm{R}$ software to conduct the calculations and modeling. The forecasting model we derived based on GM $(1,1)$ is:

$$
\hat{X}^{(1)}(t+1)=[-143.576] e^{-0.18681 t}-171.326
$$

The time response sequence we obtained based on the Grey Verhulst Model (GVM) is:

$$
\hat{X}^{(1)}(t+1)=\frac{11.07901}{0.301248+(0.097995) e^{0.399243436 t}}
$$

2) Modeling Facebook adoptions of Fortune global firms. Facebook started in 2004 but was not open up to nonstudents till September 2006. The earliest adoptions in our dataset occurred in November, 2007. Therefore, the original Facebook yearly adoption series from 2007 to 2012 is (6, 24, $49,41,49,15)$; the smoothed series becomes $(10.5,25.75$, $40.75,45,38.5,23.5)$. The forecasting model we derived based on GM $(1,1)$ is:

$$
\hat{X}^{(1)}(t+1)=[-2260.67] e^{-0.01597186 t}-2271.17
$$

The time response sequence we obtained based on the Grey Verhulst Model (GVM) is:

$$
\hat{X}^{(1)}(t+1)=\frac{-7.17146}{-0.1809+(-0.50209) e^{-0.682996363 t}}
$$

3) Modeling Twitter adoptions of Fortune global firms. Twitter entered the social media market in March, 2006. The earliest adoptions in our dataset occurred in March, 2007. Hence, the original Facebook yearly adoption series from 2007 to 2012 is $(9,21,76,24,23,7)$; the smoothed series becomes $(12,31.75,49.25,36.75,19.25,11)$. The forecasting model we derived based on GM $(1,1)$ is:

$$
\hat{X}^{(1)}(t+1)=[-240.073] e^{-0.1950287 t}-252.073
$$

The time response sequence we obtained based on the Grey Verhulst Model (GVM) is:

$$
\hat{X}^{(1)}(t+1)=\frac{-0.14489}{-0.00473+(-0.00735) e^{-0.012074208 t}}
$$

4) Comparing GM $(1,1)$ with Grey Verhulst Model. Two accuracy evaluation metrics, posterior error ratio [9] and average relative percentage error [7] were chosen to compare the performance of two grey models in forecasting social media adoption trends. Table II shows that the GM $(1,1)$ performs better than GVM does on both accuracy evaluation metrics. All three series forecasting with GM $(1,1)$ got either good or highly accurate results based on the 
posterior error ration. On the contrary, neither of three series with GVMs appeared satisfactory results in posterior error ratio.

TABLE II. PERFORMANCE METRICS: POSTERIOR ERROR RATIO \& Average Relative PERCENTAge ERROR

\begin{tabular}{|c|c|c|c|c|}
\hline \multirow{2}{*}{$\begin{array}{c}\text { Grey Series } \\
\text { Forecasting }\end{array}$} & \multicolumn{2}{|c|}{$\begin{array}{c}\text { Posterior Error Ratio } \\
\text { (C) }\end{array}$} & \multicolumn{2}{c|}{$\begin{array}{c}\text { Average Relative } \\
\text { Percentage Error (ARPE) }\end{array}$} \\
\cline { 2 - 5 } & GM(1,1) & GVM & GM(1,1) & GVM \\
\hline Youtube & $\begin{array}{c}0.2920 \\
\text { (Highly } \\
\text { Accurate) }\end{array}$ & $\begin{array}{c}0.6836 \\
\text { (Inaccurate) }\end{array}$ & $29.47 \%$ & $31.41 \%$ \\
\hline Facebook & $\begin{array}{c}0.3519 \\
\text { (Good) }\end{array}$ & $\begin{array}{c}0.8613 \\
\text { (Inaccurate) }\end{array}$ & $25.96 \%$ & $36.29 \%$ \\
\hline Twitter & $\begin{array}{c}0.3578 \\
\text { (Good) }\end{array}$ & $\begin{array}{c}1.002 \\
\text { (Inaccurate) }\end{array}$ & $36.98 \%$ & $50.55 \%$ \\
\hline
\end{tabular}

As for the three series simulated by $\operatorname{GM}(1,1)$, the Youtube series got the best result, while the Facebook and Twitter series performed a bit less accurate; however, the ratios were very close to the threshold, 0.35 , between highly accurate and good levels. Considering another metric, the Facebok series simulated with GM $(1,1)$ got the lowest average relative percentage error among the three series, followed by Youtube and Twitter series.
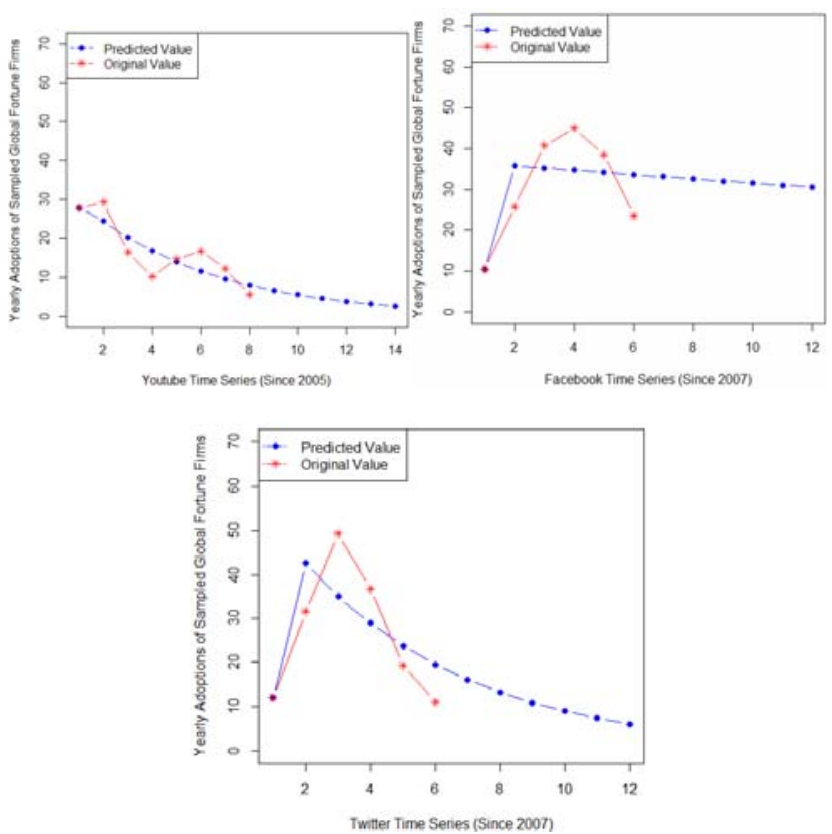

Figure 2. GM (1,1) Forecasting Yearly Trends in Top Social Media Platform Adoptions of Sampled Fortune Global Firms

Based on GM $(1,1)$ estimations, Fig. 2 plotted the actual values, predicted values, and the next six terms of predicted values of Youtube, Facebook, and Twitter adoptions by the transnational enterprises over time. It reveals that since Youtube was the pioneer of social media platform, the number of adoptions Youtube attracted in early years were greater than Facebook and Twitter. However, after joined the market for several years, Facebook and Twitter have obtained adoptions in accelerating growth and reached the peak in 2010 and 2009, respectively. In the recent two years, their adoptions started to decrease. As forecasted by GM $(1,1)$, the downward movement of Facebook adoptions would shift much slower than Twitter and Youtube does in the following six years.

\section{CONCLUSION}

Widely applied to multiple domains, grey system theory assists in modeling novel and real-time information communication systems like social media platforms with limited historical data, and thus offers flexible forecasting models for studying timing of new technology adoptions. This study utilized the APIs of top 3 social media platforms to retrieve adoption time data and demonstrated how basic grey forecasting models can be applied to modeling the trends. Although GM $(1,1)$ has much higher accuracy when compared to the grey Verhulst model in our preliminary result, it still has room for improvement. As shown in Fig. 1, Youtube adoptions declined when Facebook and Twitter adoptions rose. This suggests that we can apply Grey dynamic model GM $(1, \mathrm{~N})$ for modeling multiple variables simultaneously in the system. To determine the relationships among variables, combining grey models with the grey relational analysis [10] is likely to provide more insights in identifying significant variables in the system.

\section{ACKNOWLEDGMENTS}

The authors would like to thank the National Science Council of the Republic of China, Taiwan for financially supporting this research under Contract No. NSC 102-2410H-180 -003.

\section{REFERENCES}

[1] E. M. Rogers, Diffusion of innovations, 5th ed., New York: Free Press, 2003.

[2] C. V. Trappey and H. Y. Wu, "An evaluation of the time-varying extended logistic, simple logistic, and Gompertz models for forecasting short product lifecycles,” Advanced Engineering Informatics, vol. 22, pp. 421-430, 2008.

[3] J. Deng, “Control problems of grey system," Systems \& Control Letters, vol. 1,pp. 288-294, 1982.

[4] J. Deng, "Introduction to grey system theory," The Journal of Grey System, vol. 1, pp. 1-24, 1989.

[5] K. L. Wen and Y.F. Huang, "The analysis of population in Taiwan based on grey Verhulst method," The Journal of Grey System, vol. 8(1), pp. 27-36, 2005.

[6] C. S. Lin, "Forecasting and analyzing the competitive diffusion of mobile cellular broadband and fixed broadband in Taiwan with limited historical data,” Economic Modelling, vol. 35, pp. 207-213, 2013.

[7] E. Kayacan, B. Ulutas, and O. Kaynak, "Grey system theory-based models in time series prediction,” Expert Systems with Applications, vol. 37, pp. 1784-1789, 2010.

[8] S. Liu and Y. Lin, Grey Information: Theory and Practical Applications, London: Springer-Verlag, 2006.

[9] J. Deng, Grey System Theory, Huazhong University of Science and Technology Press, Wuhan, 1990 (in Chinese).

[10] W. Y. Wu and S. P. Chen, "A prediction method using the Grey model GMC $(1, n)$ combined with the grey relational analysis : a case study on Internet access population forecast," Applied Mathematics and Computation, vol. 169(1), pp. 198-217, 2005. 